\title{
Reliability Monitoring of GNSS Observables under the Influence of Ionospheric Disturbances
}

\author{
Kinga Węzka \\ Institute of Geodesy and \\ Geoinformation Science \\ Technical University Berlin \\ Berlin, Germany \\ Kinga.Wezka@tu-berlin.de
}

\author{
Iván Herrera-Pinzón \\ Institute for Communication and \\ Navigation \\ German Aerospace Centre \\ Neustrelitz, Germany \\ Ivan.HerreraPinzon@dlr.de
}

\author{
Roman Galas \\ Institute of Geodesy and \\ Geoinformation Science \\ Technical University Berlin \\ Berlin, Germany \\ Roman.Galas@tu-berlin.de
}

\begin{abstract}
In recent years GNSS has become the recognised core element for the provision of accurate and reliable positioning, velocity and timing (PVT) data for multiple navigational applications. However, since the occurrence of strong ionospheric disturbances impacts the accuracy of GNSS measurements, leading consequently to unreliable PVT solutions, not only precision and accuracy but also high-level reliability on the GNSS based solutions is demanded. For autonomously working GNSS receivers, it is necessary to provide algorithms responsible for the reliability control, with the ability to mitigate the influence of such phenomena. Traditionally, geometrical parameters, such as the elevation angle, and signal-related parameters, such as the C/N0, are used to describe the quality properties of GNSS observables. Nevertheless, parameters derived from physical phenomena, such as ionospheric scintillations, can provide additional information not correlated with these aforementioned approaches. The use of indices describing ionospheric scintillations is expected to provide the opportunity of improved error detection for the mitigation of threats. Thus, this work proposes the assessment of the performance of a RAIM-based reliability algorithm using a stochastic model derived from scintillation indices, for the improvement of reliability and accuracy on the final positioning solutions, in comparison with the traditional elevation angle- and C/N0 based stochastic models. Initial results indicate the potential of this approach for GNSS applications under the influence of strong ionospheric disturbances and suggest the advantages of their use.
\end{abstract}

Keywords—reliability; ionospheric disturbances; RAIM; GNSS; ionospheric scintillation

\section{INTRODUCTION}

Ionospheric disturbances cause significant degradation in the accuracy and reliability of GNSS observables and are especially harmful for multiple GNSS positioning techniques. These rapid phenomena are difficult to predict and model. Their impact on the GNSS observations is manifested as an increase of the noise level of GNSS signal, the occurrence of cycle slips, occurrence of outlier observations (blunders) and, in case of strong ionospheric scintillation events, it can even lead to unexpected loss of signal lock. The loss of signal lock is beyond the scope of the mitigation methods discussed in this work, but GNSS users should be aware that this reduces the availability of satellites and consequently weakens the geometry. In order to control the occurrence of cycle slips it is necessary to apply an efficient algorithm to detect and repair them. However, methods of reducing other scintillation effects are still the subject of discussion of many scientific papers. The most efficient approach for the reduction of ionospheric scintillation effects is the use of stochastic models which include information about the scintillation events.

The main focus of this work is the study of methods of mitigation of the influence of outlier observations caused by ionospheric scintillations. Since the presence of a blunder observation can significantly degrade the reliability of the positioning solution, it is important -especially for standalone GNSS receivers- to implement reliability monitoring tools at the user level. As a method for reliability monitoring of GNSS observations the Receiver Autonomous Integrity Monitoring (RAIM) algorithm is used. In this study the Weighted Least Square Residuals (WLSR) method [21] has been chosen. An enhancement of efficiency of RAIM under the presence of ionospheric scintillation is performed by the use of a more realistic representation of the quality of the observations.

Several stochastic models are presented as an alternative for the mitigation of unmodelled biases. Usually they are based on the satellite elevation angle, signal quality parameters (carrier-tonoise ratio) or on the least-square residuals to represent stochastic properties of GNSS observations. Although these parameters can be used as quality indicators, they do not always represent reality. The challenge is to find appropriate ways to incorporate information on unmodelled biases, such as the ionospheric scintillations, into the stochastic model. Proposed by [1], a stochastic model capable to mitigate scintillation effects uses the variance of the output error of the receiver PLL (Phase Locked Loop) and DLL (Delay Locked Loop), which expresses the quality of the range measurements. Despite the fact that the efficiency of this model is very promising, it is also worth to notice that users have limited access to use those parameters. This study aims for a more realistic representation of the GNSS observations quality during the occurrence of strong ionospheric scintillations. Thus, the first proposed model implies the use of the widely known ionospheric scintillation indices $\left(S_{4}\right.$ and/or $\left.\sigma_{\phi}\right)$. An alternative to this model it is proposed to take into consideration the scintillation index together with the satellite elevation angle. Both, scintillation indices and elevation angles are relevant to the quality of GNSS observations. They describe 
different sources of GNSS errors degrading quality of observations, which guaranties that they are uncorrelated. The lack of correlation between these parameters suggests combining them to obtain a better representation of the quality of observations.

In order to explore the performance of the proposed stochastic model, the rest of the paper is organised as follows. Section II discusses the methodology used in these experiments, describing the methods to calculate scintillation indices and providing the description of the GNSS observation models and their corresponding stochastic models. Furthermore, an essential description of the reliability theory applied to integrity monitoring is presented. Then, in section III, a description of the experimental setup and the analysis of obtained results are presented. Finally, section IV discusses these results and presents an outlook for future activities.

\section{MethodOLOGY}

\section{A. Ionospheric scintillations}

The presence of ionospheric irregularities causes random and rapid electron density fluctuation. These irregularities diffract radio waves to cause amplitude and phase scintillation of satellites radio signals [2] [22] [23]. Thus, GNSS signals affected by scintillations can be describe as [14]:

$$
E=A e^{j \phi}=E_{0} \delta E=\left(A_{0} \delta A\right) e^{j\left(\phi_{0}+\delta \phi\right)}
$$

Where $E_{0}=A_{0} e^{j \phi_{0}}$ is the nominal signal without scintillation effects, with nominal amplitude $A_{0}$ and nominal phase $\phi_{0}$. While the scintillation-affected signal is represented by $\delta E=\delta A e^{j \delta \phi}$ with amplitude $\delta A$ and phase $\delta \phi$ scintillations.

Both, amplitude- and phase scintillations pose a serious threat for the performance of GNSS based positioning systems. Phase scintillations induce an unexpected frequency Doppler shift in the signal carrier. If the shifts exceed the bandwidth in the GPS receivers' phase lock loops (PLL), the signal is lost and must be required again [5] [6]. Amplitude scintillations cause signals to fade below the average level. When the depth of fading exceeds the fade margin of a receiver, the signal becomes buried in noise and signal loss and cycle slips are encountered [5] [6]. To represent the intensity of ionospheric scintillations in GNSS signals, two scintillation indices are commonly used: phase scintillation index $\left(S_{4}\right)$ and amplitude scintillation index $\left(\sigma_{\phi}\right)$.

The amplitude scintillation index $S_{4}$ is computed from signal power $P$, or signal intensity. To calculate the signal power, inphases and quadrature-phases components from the correlator output recorded at $50 \mathrm{~Hz}$ sampled rate are used. Then, the amplitude scintillation index $S_{4}$ is defined as a normalized variance of the signal total power $S_{4 T}$ minus predicted amplitude scintillation index due to the effects of ambient noise $S_{4 N o}[11]:$

$$
\begin{aligned}
& S_{4}=\sqrt{S_{4 T}-S_{4 N o}} \\
& =\sqrt{\frac{E\left\{P^{2}\right\}-(E\{P\})^{2}}{(E\{P\})^{2}}-\frac{100}{S / N o}\left[1+\frac{500}{19 S / N o}\right]}
\end{aligned}
$$

Where, $E\{\cdot\}$ denotes the expected value calculated over predefined window of time and $S / N o$ is the signal-to-noise density.

The phase scintillation index $\left(\sigma_{\phi}\right)$ is calculated from the carrier phase measurements sampled at $50 \mathrm{~Hz}$. However, the total carrier phase recorded by GNSS receiver contains also a lowfrequency components including effects of the satellite-to-user dynamic, the slowly varying receiver clock, atmospheric effects, satellite clock and low-frequency multipath. Thus, prior to the computation of the scintillation index, low-frequency components must be separated from the recorded carrier phases. This process is performed with a $6^{\text {th }}$-order high-pass Butterworth filter. In practice, this $6^{\text {th }}$ order filter is implemented by three cascaded $2^{\text {nd }}$-order filters to increase the stability of the filter response. The cut-off frequencies, to detrend raw carrier phase measurements, is set up to $0.1 \mathrm{~Hz}$ [11].

The phase scintillation index $\sigma_{\phi}$ is characterised by its standard deviation of the detrended phase $\delta \phi$ of the L1 signal, which can be written as:

$$
\sigma_{\phi}=\sqrt{E\left\{\delta \phi^{2}\right\}-(E\{\delta \phi\})^{2}}
$$

Where $E\{\cdot\}$ denotes expected value calculated over a predefined window of time (set up to $1 \mathrm{~s}$ ) and $\delta \phi$ is the detrended carrier phase containing scintillation signatures.

Ionospheric scintillations mainly occur at high latitude (close to the auroral and polar cap regions) and in the equatorial band that extends from about $20 \mathrm{~S}$ to $20 \mathrm{~N}$ of the magnetic equator. The processes that produce scintillations in those two regions are quite different and cause differences in the characteristics of scintillations.

\section{B. GNSS Positioning methods and stochastic models}

To study scintillation effects on GNSS positioning, GNSS data are processed using a Single Point Positioning (SPP) algorithm to evaluate the positioning error and reliability of the obtained solutions. The SPP algorithm is a standalone positioning technique using code-phase observations from a single GNSS frequency. To estimate the user position, the Weighted Least Square (WLS) method is applied.

The linearized observation equation for this method is:

$$
y=A x+e
$$

which corresponds to a general form of a Gauss-Markov functional model [9][15][18]:

$$
E\{y\}=A x, \quad E\{e\}=0
$$

The stochastic model describes the dispersion matrix of the observations and defines the variance-covariance matrix $Q_{y}$ is expressed as follow:

$$
D\{y\}=E\left\{e e^{T}\right\}=Q_{y}=\sigma_{0}^{2} G_{y}
$$

Where $D\{\cdot\}$ denotes dispersion operator and $E\{\cdot\}$ denotes the expectation operator, $y$ is the $n \times 1$ observation vector; $x$ is the $m \times 1$ unknown parameter vector; $A$ is the $n \times m$ coefficients matrix with $n>m$; $e$ is the normally distributed $n \times 1$ random error vector (unknown) $e \sim \mathcal{N}\left(0, \sigma_{0}^{2} G_{y}\right) ; \sigma_{0}^{2}$ is the $a$ 
priori variance of unit weight, often assumed to be $1 ; G_{y}$ is the $n \times n$ symmetric positive-definite cofactor matrix of observation;

For the model described in equation (4) the least-squares estimator is defined as:

$$
\hat{x}=\arg \min _{x}\|y-A x\|_{Q_{y}}^{2}, \quad x \in \mathbb{R}
$$

If the inverse of variance-covariance matrix is used as a weight, the best linear uniformly unbiased estimator of estimated is given by:

$$
\hat{x}=\left(A^{T} Q_{y}^{-1} A\right)^{-1} A^{T} Q_{y}^{-1} y
$$

The result of GNSS positioning model $(\hat{x})$ contains the increments for coordinates and receiver correction, thus, the estimate of the unknown user coordinates is obtained by adding the incremental component to the linearization point.

\section{Stochastic Propertis of GNSS Observations}

The most frequent GNSS data processing strategy for the positioning solution considers a stochastic model based on a weight matrix. If errors of the measurements are assumed to be uncorrelated and their precisions are the same, the measurement weight matrix of observations can be represented as the inverse of the covariance matrix $W=Q_{y}^{-1}$. Where the diagonal elements of $Q_{y}$ are variances of the individual GPS observations $\sigma^{2}$. Those variances can be obtained based on the a priori variance $\sigma_{0}^{2}$ (unit weight) and the adequate weight $w$ using $\sigma^{2}=\sigma_{0}^{2} / w$. While the cofactor values are given by $q=1 / w$. The weights $w$ are representing the reciprocal of each variance, $\sigma^{2}$, and they are expressed as $w=1 / \sigma^{2}$. The simplified form of weight matrix $W$ is a diagonal matrix:

$$
W=\operatorname{diag}\left(w_{1}, w_{2}, w_{3}, \ldots w_{n}\right)
$$

Traditional stochastic models use geometrical- (satellite elevation angle) or signal quality (carrier-to-noise ratio) parameters in order to describe properties of GNSS observables. Since the carrier-to-noise ratio is a key parameter analysing the performance of GNSS receivers [16], many of the stochastic models are based on it. These models are mostly applied to mitigate the multipath effect. As a benchmark, this study implements a $\mathrm{C} / \mathrm{No}$ - dependent stochastic model, called SIGMA- $\varepsilon$ [13], describing the carrier phase variance given in $\mathrm{mm}^{2}$ as the function of $\mathrm{C} / \mathrm{No}$, represented by:

$$
\sigma_{\phi}^{2}(C / N o)=C_{i} \cdot 10^{-\left(\frac{C / N o}{10}\right)}
$$

Thus, the weight can be written as:

$$
w(C / N o)=\frac{1}{\sigma_{\phi}^{2}(C / N o)}
$$

Where $C / N o$ denotes carrier-to-noise ratio parameters and $C_{i}$ is the factor, given in $m^{2} \mathrm{~Hz}$, which depends on the carrier loop noise bandwidth and wavelength. The value of $C_{i}$ which was estimated based on analysing multiple data sets is equal to $1.61 \times 10^{-4} \mathrm{~m}^{2} \mathrm{~Hz}$ and it can be used as a constant [8].
The second parameter, used for description of the stochastic properties of GNSS observations, is the satellite elevation angle. The basic assumption is that observations at low elevation angles are noisier than at high elevation. One of the most frequently used models is [10][20]:

$$
w(e l)=\sin ^{2}(e l),
$$

Those models do not include information about atmospheric conditions. Since quality of the GNSS observations depends strongly on the sudden disturbances in the ionosphere, such as the occurrence of strong ionospheric scintillations, it is advisable also to incorporate this information.

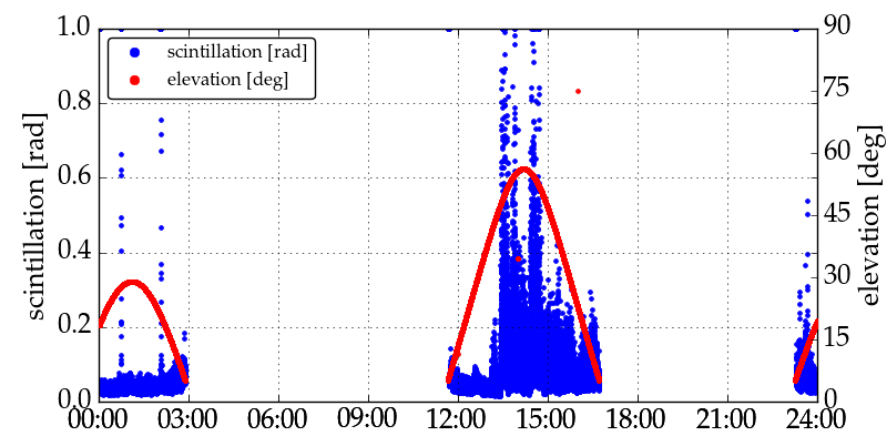

Fig. 1. Phase scintillation and satellite elevation angle measured at station Kiruna/Sweden (March 17th, 2015) for satellite PRN 15

Fig. 1 shows the uncorrelated behaviour of the scintillation and satellite elevation angle and implies the unrealistic assumption made by elevation-dependent weighting models, namely, the larger the satellite elevation angle, the better the observation quality, and the smaller the observation variance.

As it was aforementioned, this study addresses the modelling of ionospheric scintillations using the signal scintillation indices. Since ionospheric scintillations are rapid variations in the amplitude and phase of GNSS signals, it is expected that they also relate to the signal quality. For those reasons two stochastic models for the weights of observations, based on ionospheric scintillation indices, are proposed here. Firstly:

$$
w\left(S_{i d x}\right)=1+a \cdot \exp ^{-S_{i d x}}
$$

The weights are calculated with the use of equation (13) based on the scintillation index $S_{i d x}$ and an empirical constant $a$. The constant $a$ has been introduced to avoid singularities. Its value has been estimated based on a larger time series and is taken as 0.6 .

The second approach relies on applying of the scintillation index and of the elevation angle together. This model based on the assumption that phase scintillation index represents only high frequency noise. For this reason this works suggests to combine it with the use of other parameter describing low frequency noise. This could be realized by adding an elevationdependent term to the model. The combine weighted function is therefore written as:

$$
w\left(S_{i d x} / e l\right)=\sin ^{2}(e l)+a \cdot \exp ^{-S_{i d x}}
$$

Fig. 2 shows the relations between the proposed weight functions. The upper plot shows the weight calculated using the 
eq. (12). The second plot shows weights calculated with the model of eq. (11). It is clearly seen that the C/N0 weighting model reflects some disturbances in the high elevation angles. However, two other models - eq. (13) and eq. (14) - seem to reflect ionospheric scintillations more appropriately. It is expected that those features will increase the ability of the reliability algorithms to detect and mitigate the anomalous observations.

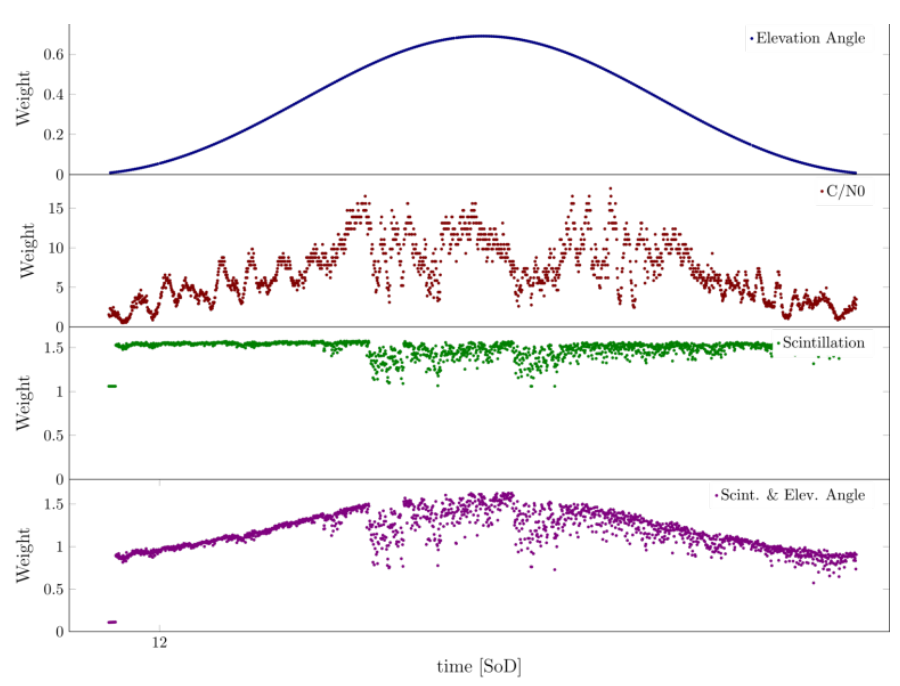

Fig. 2. Comparison of the applied weight values for satellite PRN 15. C/N0-dependent weight values, elevation-dependent weight values, scintillation-dependent weight values and scintillation and elevation-dependent weight values

\section{Realiability monitoring related issues}

The reliability of the navigation system refers to its ability to perform and maintain its functions in routine circumstances, as well as unexpected circumstances for a certain period of time. This parameter is used to determine the confidence level for the observations used during the position calculation. The reliability theory for quality evaluation of data was first introduced by Baarda [3], and it was applied to reliability control of the geodetic networks. Since then, Baarda's concept has been also adapted and used in many procedures/schemes to control the reliability of GNSS measurements. This is firmly based on internal reliability defining the capability of a system to detect outlier in any given observation, and external reliability which define the maximum effect of any undetected outlier on the estimated parameters.

The reliability assessment can be conducted through the use of integrity tests. To meet this expectation, the RAIM (Receiver Autonomous Integrity Monitoring) technique is applied at the user level to check the consistency of the determined solutions. Traditional RAIM techniques are composed of the following main elements [7]: Fault Detection and Exclusion (FDE) and optionally Protection Level (PL) computation. In this study the Least Square Residual (LSR) method for reliability monitoring has been considered [17]. The obtained result from a single epoch solution (snapshot) allows unequivocal identification of blunder observations. Moreover, the Weighted Least Square Method (WLSM) allows improving both accuracy and integrity of the position solution by including of individual weighting of the code-phase measurements [21].

When the general linearized model (4) and the assumption that the condition of redundant observations have been fulfilled, the estimated weighted least-square residuals of the pseudo-ranges can be obtained from the least square fit as:

$$
\hat{e}=\hat{y}-y=A \hat{x}-y
$$

For covariance of estimated parameters, being equal, $D\{\hat{x}\}=$ $Q_{\hat{x}}=\left(A^{T} Q_{y}^{-1} A\right)^{-1}$ the covariance of estimate residuals is expressed as:

$$
Q_{\hat{e}}=Q_{y}-A\left(A^{T} Q_{y}^{-1} A\right)^{-1} A^{T}
$$

The normalized residuals can be written as:

$$
z_{i}=\left|\frac{\hat{e}_{i}}{\sqrt{\left\{Q_{\hat{e}}\right\}_{i i}}}\right|, \quad i=1,2 \ldots n
$$

where $n$ denotes the number of observations and $\left\{Q_{\hat{e}}\right\}_{i i}$ is the ith diagonal element of the cofactor matrix.

The squared weighted norm of error estimates, called weighted sum of the squared errors (WSSE), describes a scalar measure for inconsistency of the linear system and can be written as follows:

$$
W S S E=\hat{e}^{T} Q_{y}^{-1} \hat{e}
$$

The WSSE plays the role of the basic observation in RAIM algorithms. It describes the magnitude of discrepancy (mismatch) between the observation and the measurement. Thus we can use $\sqrt{W S S E}$ as the test statistic in order to judge the goodness of the least square fit [21].

Before starting the statistical tests the performance thresholds must be specified in terms of probability of false alarm $\alpha$ and probability of missed detection $\beta$. According to the Baarda's procedure, the risk level of global test $\alpha$ must be related to the risk level of local test $\alpha_{0}$. Furthermore, those parameters should be related to probability of missed detection for both tests, with assumption that $\beta=\beta_{0}$. The interrelation of those parameters can be expressed as [3]:

$$
\begin{aligned}
\lambda & =\lambda_{0}{ }^{2} \\
\chi_{\beta, n-m, \lambda_{0}}^{2} & =\chi_{1-\alpha, n-m}^{2}
\end{aligned}
$$

Whit $\lambda$ the non-centrality parameter of the biased non-central $\chi^{2}$ distribution related to global test. The relations written above indicate that only two of mentioned parameters $\left(\alpha, \alpha, \beta=\beta_{0}\right)$ can be chosen arbitral. For selected $\alpha_{0}, \beta_{0}$ the parameter $\alpha$ can be calculated based on the above equations. When defining a value for the selected parameter it is necessary to consider that the large value $\alpha_{0}$, implies a smaller threshold of the local test, causing exclusion of a higher number of correct observations. Furthermore, the large value of $\beta_{0}$ causes higher probability of missed detection, therefore more erroneous observation will be accepted as correct ones. 
To perform a statistical reliability testing/outlier detection procedure global and local integrity tests are applied. The global test is used for assessing whether the set of measurements includes errors or not and it is performed by testing of the test statistic value against threshold value.

The test statistic $T_{G}=\sqrt{W S S E}$ has a $\chi^{2}$-square distribution with n-4 degrees of freedom (assuming that the measurement errors are independent and normally distributed with zero mean) [17] and $\chi^{2}$ distribution is central under $H_{0}$ and noncentral under $H_{a}$. Then the hypotheses in global test are tested as:

$$
\begin{aligned}
& H_{0}: T_{G} \leq \chi_{(1-\alpha, n-m)}^{2} \text { - reliable observations } \\
& H_{a}: T_{G}>\chi_{(1-\alpha, n-m)}^{2}-\text { unreliable observations }
\end{aligned}
$$

The null hypothesis $H_{0}$ states that the model is correct and there are no bias errors in the set of measurements, it means that the solution is concluded to be reliable. If test statistic exceeds the threshold, the null hypothesis is rejected, an inconsistency is assumed and the local test should be performed.

The local test is performed to identify which measurement is the cause of the inconsistency. As a test statistic, $T_{L}$, normalized residuals (17) are used. The hypotheses are tested against a quantile of a normal distribution with predetermined false alarm rate $\alpha_{0}$ value to identify outliers:

$$
\begin{aligned}
& H_{0, i}: T_{L} \leq N_{1-\frac{\alpha_{0}}{2}}-\text { no outlier presents } \\
& H_{a, i}: T_{L}>N_{1-\frac{\alpha_{0}}{2}} \text { - outlier presents }
\end{aligned}
$$

If the global test fails, and null hypothesis of local test is accepted, the local test does not indicate an erroneous measurement in the observation set and obtained solution is concluded to be unreliable. The alternative hypothesis of local test $H_{a, i}$ denotes that the model is wrong, or that the assumption is not correct. It means that at least one error which is not zero-mean normally distributed exist in the set of measurements. Thus k-th observation is suspect to be an outlier when:

$$
H_{a, k}: z_{k}>z_{i} \forall_{i} \cap z_{k}>N_{1-\frac{\alpha_{0}}{2}}
$$

If the local test detects an erroneous measurement, then this observation is rejected and the remaining set of observations is used to calculate the solution. The process can be repeated until no more errors are detected or until the condition of redundant observation is not fulfilled. A diagram of the sequence actions of the RAIM algorithm is presented in Fig. 3.

\section{NUMERICAL FINDINGS}

\section{A. Experimental Setup}

Initial investigations have been conducted using time series of GNSS observations gathered at high-latitude $\sim 67.5 \mathrm{~N}$ (Kiruna/Sweden). The positioning solution was performed using $1 \mathrm{~Hz}$ GPS observations. While, the analysis of ionospheric scintillation indices were calculated using $50 \mathrm{~Hz}$ GPS (carrier-phase observations and accumulated in-phases and quadrature-phases from the correlator output). This sampling rate is necessary to avoid of loss of high-frequency components of phase variation, which contains the phase scintillation information.

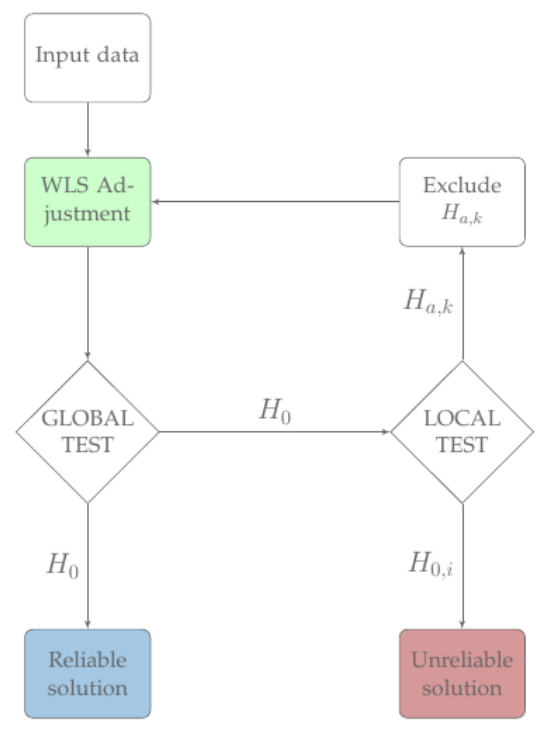

Fig. 3. Flow chart for a RAIM-like algorithm

Since ionospheric perturbations are directly associated with geomagnetic storms, the initial identification of those phenomena has been performed with the use of the planetary Kp-index. This index represents irregular disturbances of the geomagnetic field caused by solar particle radiation [4]. Fig. 4 shows planetary Kp-index values for the day 17 of March of 2015.

Planetary geomagnetic indices $\mathrm{Kp}$ greater than 4 are an indication of strong geomagnetic activity, and ionospheric scintillations are more likely to occur [19]. Based on this premise, the experiments were undertaken during periods the most ionospherically disturbed - on March 17, 2015 (St. Patrick's Day ionospheric storm).

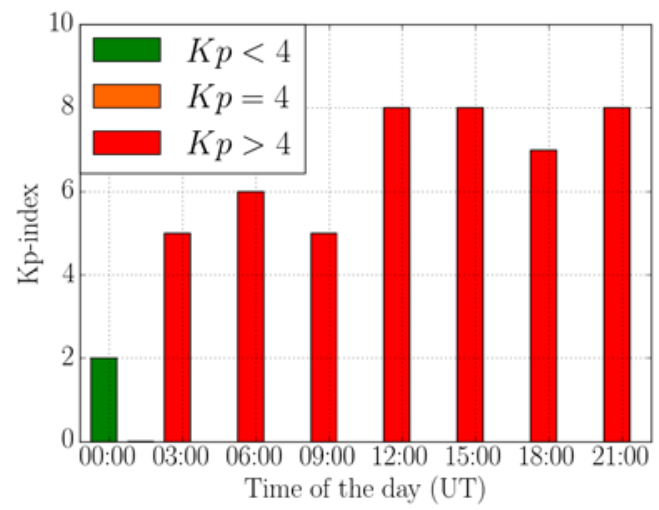

Fig. 4. Planetary Kp-index on 17 March 2015

Since at high latitudes strong phase scintillations and weak amplitude scintillations are frequently observed, for the remaining part of the analysis only phase scintillation indices will be used [12]. To obtain information about scintillation, synchronous in time to the GNSS observations, the phase scintillation index was calculated with a frequency of $1 \mathrm{~Hz}$. 


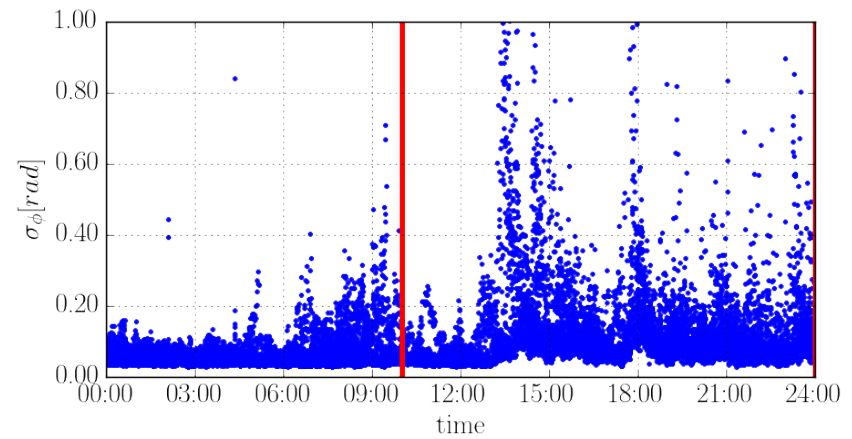

Fig. 5. Phase scintillation indices obtained from all satellites on 17 March 2015

To investigate the accuracy and reliability of the positioning solution the Single Point Positioning (SPP) method with a RAIM algorithm was used. The SPP method is based on the use of L1 C/A code observations. To reduce biases caused by ionosphere and troposphere Klobuchar and Saastamoinen models were used respectively. The weighted least-square (WLS) method and RAIM algorithm described in the section II-C have been used. The following performance thresholds for the statistical reliability testing have been specified:

- the probability of false alarm $\alpha=5 \%$

- the probability of missed detection $\beta=20 \%$

\section{B. Results}

The evaluation of the efficiency of the proposed stochastic models within the process of reliability control of GNSS observations, collected during the occurrences of strong ionospheric scintillations, is performed using the scenarios presented in the Table 1.

Table 1. GNSS processing scenarios for the evaluation of the efficiency of stochastic models

\begin{tabular}{c|c|c}
\hline Scenario & Stochastic model & RAIM \\
\hline WLS-1 & Elevation Angle dependent & No \\
\hline WLS-2 & C/N0 dependent & No \\
\hline WLS-3 & Scintillation dependent & No \\
\hline WLS-4 & $\begin{array}{c}\text { Scintillation \& Elevation Angle } \\
\text { dependent }\end{array}$ & No \\
\hline WLS+RAIM-1 & $\begin{array}{c}\text { Elevation Angle dependent } \\
\text { WLS+RAIM-2 }\end{array}$ & Yes \\
\hline WLS+RAIM-3 & $\begin{array}{r}\text { Scintillation dependent } \\
\text { WLS+RAIM-4 }\end{array}$ & Yes \\
\hline Scintillation \& Elevation Angle & Yes \\
\hline
\end{tabular}

These processing scenarios are divided in two groups of solutions. First group gathers the results obtained from weighted least square (WLS) with the weight matrix levering the quality of the observations, although no RAIM algorithm is enabled, including scenarios WLS-1 to WLS-4. Results are limited to the time window where the strongest ionospheric scintillations were observed (Fig. 5), starting at $10 \mathrm{~h}$ UTC and going until the end of the day. Fig. 6 shows the differences between reference coordinates of the site (with a higher expected accuracy as they are calculated with more precise geodetic techniques in the absence of disturbances) and the solutions obtained for each scenario.

The second group of scenarios incorporates the use of the WLS method with different stochastic models befitted with a RAIM algorithm. This group includes the scenarios WLS+RAIM-1 to WLS+RAIM-4. Once again, results are limited to the time window from $10 \mathrm{~h}$ UTC to $24 \mathrm{~h}$ UTC. Figure 7 shows the differences between reference coordinates and the solutions calculated for each scenario.

Fig. 6 shows that the C/N0 (WLS-2) and scintillation (WLS-3) based solutions reflect a fairly realistic trend than those based on elevation angle (WLS-1) and elevation angle \& scintillation (WLS-4). However the behaviour of the $\mathrm{Z}$ component between $17 \mathrm{~h}$ and $18 \mathrm{~h}$ (in oval) shows a larger discrepancy between the solutions obtained from scenario WLS-2 and WLS-3. This is caused by the presence of strong signal scintillations during this period of time (see Fig. 5).

In fact, the analysed data set does not show many significant effects on C/A code, such as sudden disturbances caused by ionospheric scintillation. This is mainly due to the fact that code-phase observations are less degraded by this phenomenon than carrier-phase ones [19]. However, the presence of large outliers during these epochs requires the use of a RAIM algorithm capable to perform the reliability control of the final solutions.

Additionally, results shown in the Fig. 7 represent the solutions obtained from the weighted least-square process with the RAIM algorithm enabled. The results displayed on Fig. 7 with respect to those of Fig. 6 confirm the well-known fact that the use of a RAIM algorithm leads to an improvement on the positioning solution. This is reasserting by the behaviour of the mean error values presented in Table 2.

Comparison of the errors between solutions from different stochastic model confirms that solutions from scenarios WLS+RAIM-1 and WLS+RAIM-4 are worse than solutions from scenario WLS+RAIM-2 and WLS+RAIM-3. This is explained by the assumption of elevation-dependent variance models which state that the higher elevation angles the better quality of observations. Thus, they become inefficient for measurements which are strongly affected by some sudden disturbances which occur for high elevation angle. However, the approach which takes into account both effects, the satellite elevation angle and the ionospheric scintillation index, points a bigger number of unreliable solutions than the one incorporating only elevation angle. While, the mean error values obtained for both scenarios are quite similar. 


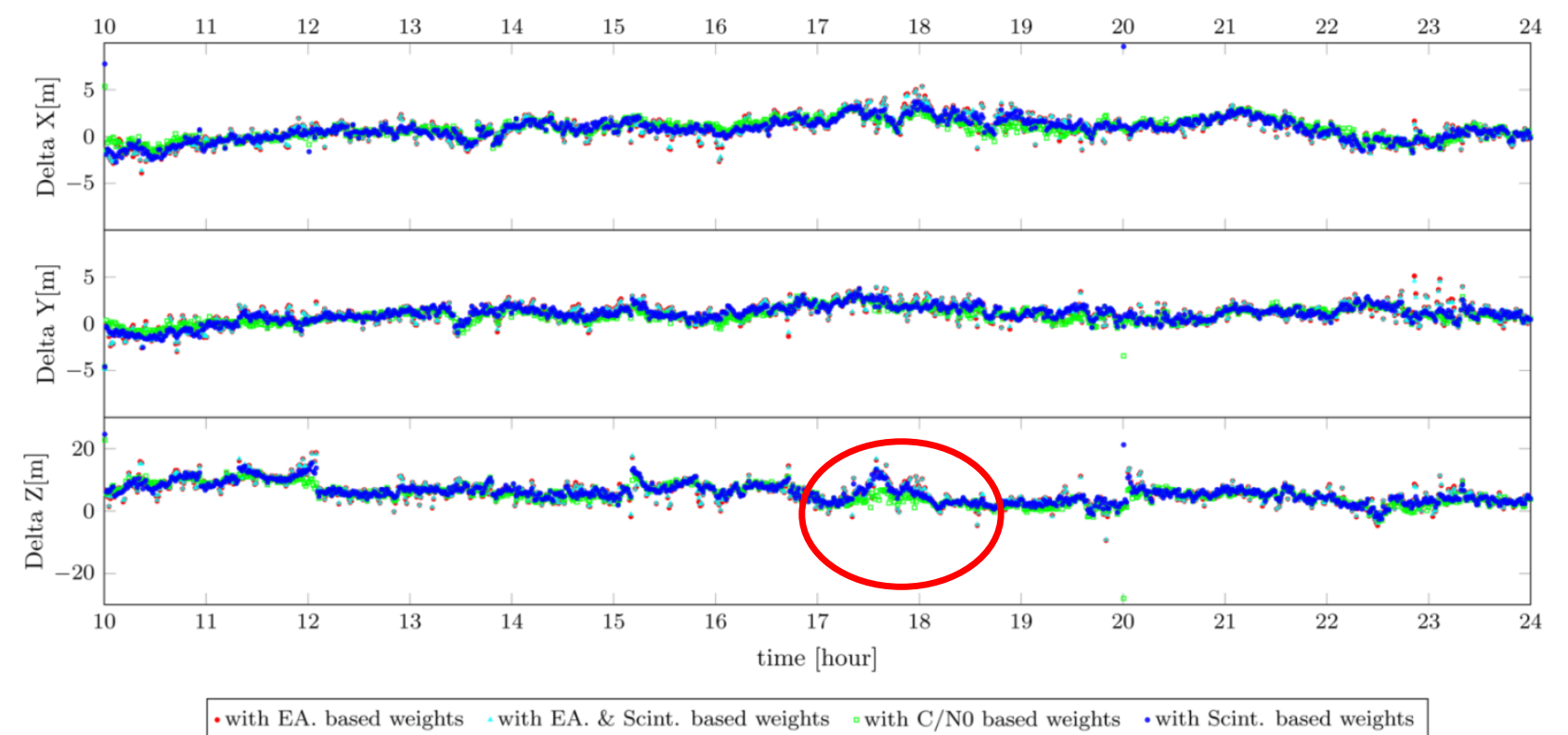

Fig. 6. Time series of the weighted least-square solution

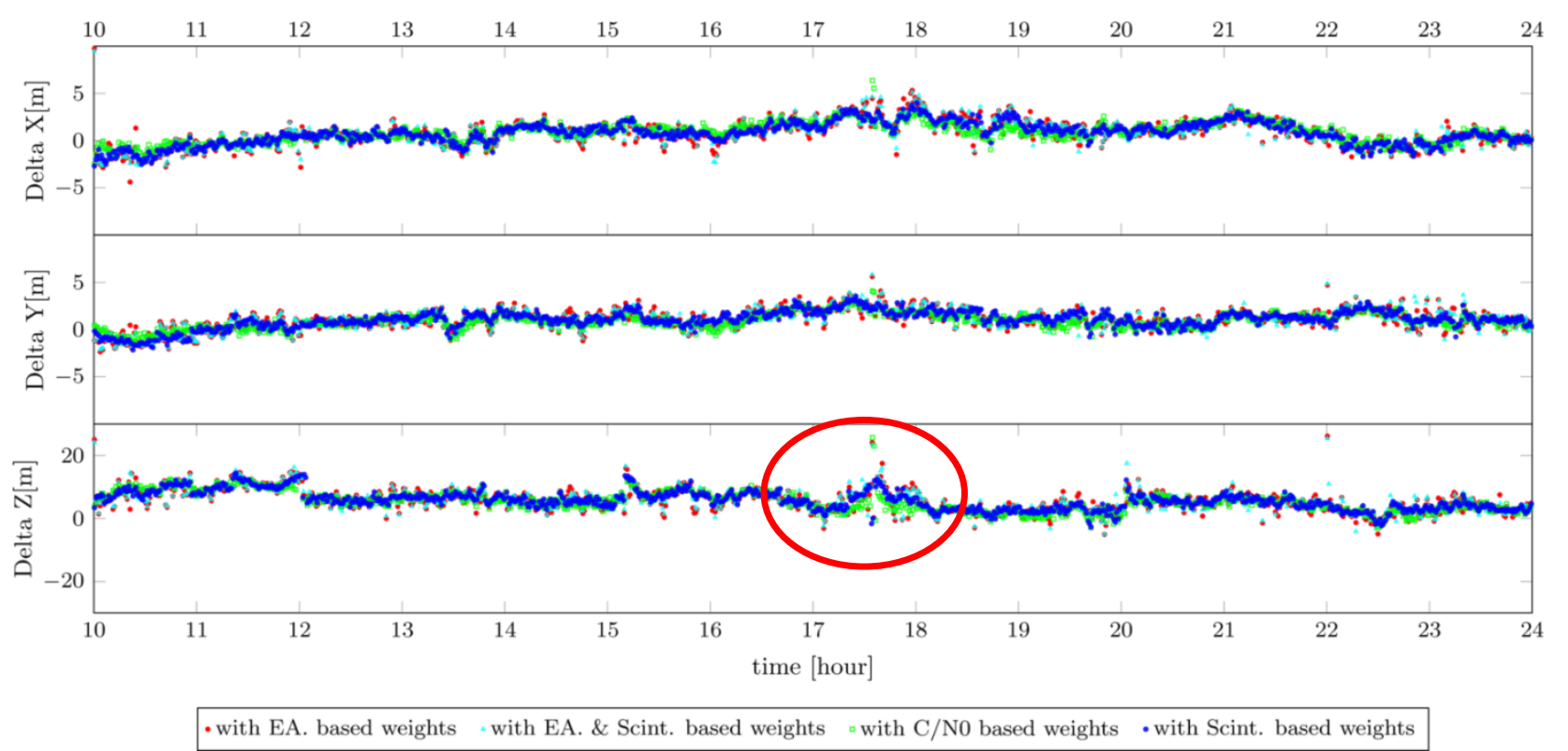

Fig. 7. Time series of the weighted least-square with RAIM solution

Results of mean error displayed on Table 2. point to a slightly better performance for solutions with the using of the C/N0 parameter (WLS+RAIM-2) than those with the scintillation parameter (WLS+RAIM-3). However the number of unreliable solutions (856) is much bigger for the solutions with weights based on C/N0 values (see Table 4). This is likely due to a frequent appearance of sudden drops in $\mathrm{C} / \mathrm{NO}$ based weights and may result in a misrepresentation of the quality of the measurements when this parameter is used as a quality indicator. Furthermore, using the unrealistic weight values can lead to achieve the threshold of falls alarm and consequently cause rejection of the correct observations. In contrast, the number of unreliable solutions of the approach with weights based on the scintillation index indicates that more solutions passed the reliability test. Thus, on this basis it is possible to affirm that the use of a stochastic model incorporating scintillation parameters leads to a more realistic description of the data quality gathered in the presence of strong ionospheric scintillations.

The maximum error values presented in Table 3 reaffirm the validity of the use of RAIM algorithms for the observations gathered in the presence of ionospheric scintillation. It can be clearly seen that the extremely large values of RMS are significantly reduced. Once again the best results are noticed for scenarios WLS+RAIM-2 and WLS+RAIM-3. 
Table 2: Mean Error [m] per component, with respect to the reference position

\begin{tabular}{c|c|c|c}
\hline \multirow{2}{*}{ Scenario } & \multicolumn{3}{|c}{ RMS } \\
\cline { 2 - 4 } & $\mathbf{X}$ & $\mathbf{Y}$ & $\mathbf{Z}$ \\
\hline WLS-1 & 1.69 & 1.52 & 6.98 \\
\hline WLS-2 & 1.36 & 1.23 & 6.22 \\
\hline WLS-3 & 1.44 & 1.38 & 6.60 \\
\hline WLS-4 & 1.67 & 1.49 & 6.98 \\
\hline WLS+RAIM-1 & 1.53 & 1.45 & 6.57 \\
\hline WLS+RAIM-2 & 1.31 & 1.21 & 6.10 \\
\hline WLS+RAIM-3 & 1.37 & 1.36 & 6.43 \\
\hline WLS+RAIM-4 & 1.51 & 1.44 & 6.60 \\
\hline
\end{tabular}

Table 3: Maximum Error [m] per component, with respect to the reference position

\begin{tabular}{c|c|c|c}
\hline \multirow{2}{*}{ Scenario } & \multicolumn{3}{|c}{ Max. RMS } \\
\cline { 2 - 4 } & $\mathbf{X}$ & $\mathbf{Y}$ & $\mathbf{Z}$ \\
\hline WLS-1 & 35.25 & 16.06 & 77.47 \\
\hline WLS-2 & 15.51 & 14.18 & 39.45 \\
\hline WLS-3 & 16.51 & 11.10 & 36.72 \\
\hline WLS-4 & 34.62 & 16.88 & 75.29 \\
\hline WLS+RAIM-1 & 19.72 & 6.36 & 39.71 \\
\hline WLS+RAIM-2 & 10.86 & 4.67 & 29.26 \\
\hline WLS+RAIM-3 & 10.98 & 5.38 & 29.45 \\
\hline WLS+RAIM-4 & 19.56 & 6.31 & 39.45 \\
\hline
\end{tabular}

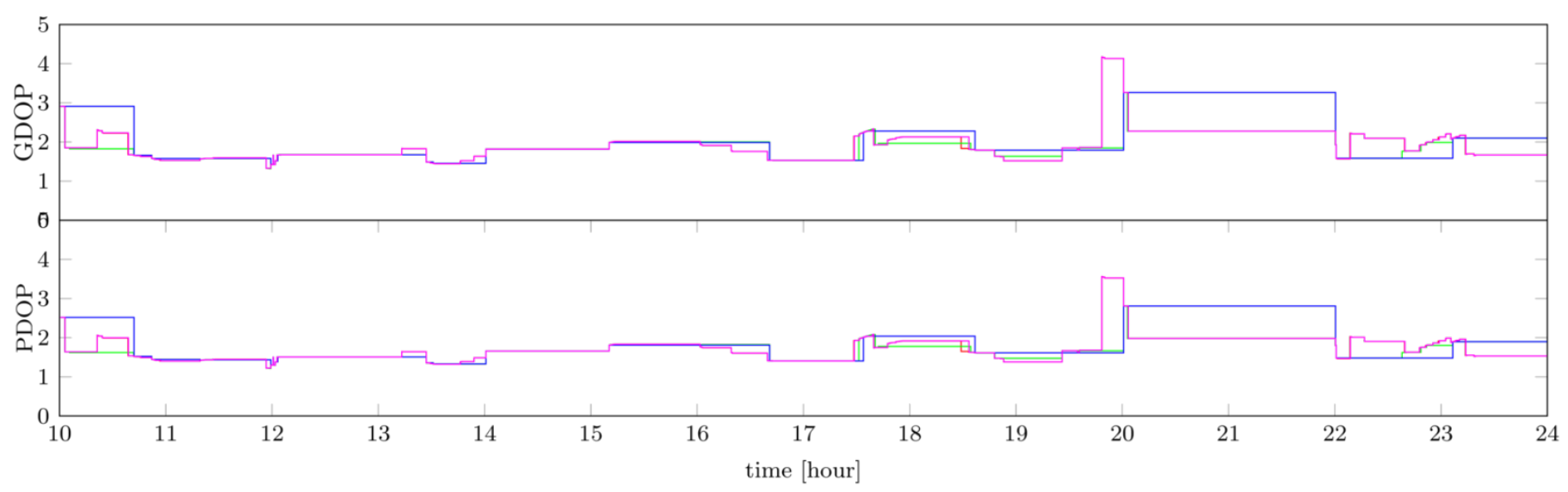

— with EA. based weights —- with C/N0 based weights —- with Scint. based weights — with EA. \& Scint. based weights

Fig. 8. GDOP and PDOP of the weighted least-square with RAIM solution

Since RAIM algorithms a prone to reject blunder observations, a drop on the number of satellites and therefore a poor geometry of the final solution is expected. Thus, it is also advisable to monitor the parameters describing dilution of precision (DOP) of the obtained results. The two selected parameters represent the geometric (GDOP) and 3D positions (PDOP) values of the DOP for solutions when the RAIM algorithm was used are displayed in Fig. 8. It is generally accepted that the value of these parameters should be lower than six. Thus, all the obtained results in these experiments meet this expectation.

The total number of rejected observations during the time window of the study can be considered as an indicator of the detection capability of the applied RAIM algorithm. Table 4 shows the results of this indicator, together with the number of unreliable solutions for the same period of time. The solution has unreliable status if the global test fails and the local test is not able to indicate an erroneous observation. The comparison between the two solutions, RAIM solution incorporating scintillation weighted function (WLS+RAIM-3) and RAIM solution incorporating C/N0 weighted function (WLS+RAIM-
2), shows that the number of rejected observations for the solution type WLS+RAIM-2 is almost 2 times higher than for solution type WLS+RAIM-3, while the number of rejected observations is almost 8 times higher. In terms of real-time navigation solutions, the smaller number of unreliable solution is usually more important than higher number of rejected satellites. This fact can indicate that the results obtained by using the scintillation index as a quality indicator parameter fits slightly better to the condition in which the data was collected. It is also noticed that the highest number of rejected satellites was observed for solutions using the elevationdependent model WLS+RAIM-1 while the number of unreliable solution is really low. Nevertheless, error values presented in Table 2 and Table 3 are not satisfactory for this approach. 
Table 4: Number of unreliable solutions and rejected satellites

\begin{tabular}{c|c|c}
\hline Scenario & $\begin{array}{c}\text { \# of unreliable } \\
\text { solutions }\end{array}$ & $\begin{array}{c}\text { \# of rejected } \\
\text { observations }\end{array}$ \\
\hline WLS+RAIM-1 & 220 & 2378 \\
\hline WLS+RAIM-2 & 856 & 1821 \\
\hline WLS+RAIM-3 & 129 & 979 \\
\hline WLS+RAIM-4 & 188 & 1821 \\
\hline
\end{tabular}

An overview of the obtained results is presented in Figure 8 and Figure 9, with the histograms of the distribution of the positioning error (PE) for each one of the methods. The histograms in Fig. 9 show the distribution of positioning error for weighted least square solutions without RAIM, while histograms in Fig. 10 show the distributions of PE for weighted least square solutions with RAIM. These results confirm that applicability of RAIM algorithm enhance the quality of the results and ensure the more reliable positioning solution. Moreover, the approach incorporating scintillation index as a quality parameter to define stochastic model show a marginal better performance than the one with using $\mathrm{C} / \mathrm{No}$ as a quality indicator.

\section{DISCUSSION}

This paper discusses reliability monitoring for GNSS observations gathered in the presence of ionospheric disturbances, such as ionospheric scintillations. There is no doubt that stochastic models play a key role in outlier detection and integrity monitoring. The use of erroneous quality indicators tend to overestimate the obtained results and consequently increase the number of the rejected observations. Therefore, this work devoted efforts on the possibility of using information regarding ionospheric scintillations for the definition of a more realistic description of data quality. A simple, yet efficient, way of incorporating scintillation index into stochastic model for the reliability monitoring process is described. First initial results are very promising and demand that further and more detailed studies.

Obtained results lead to the conclusion that the use of parameter of satellite elevation angle does not accurately describe the data when the sudden disturbances are noticed. This can be especial important in the process of reliability control and for the detection of outlier observations. The analysis showed that the precision of the coordinates becomes better with the introduction of the models using the C/No and scintillation parameters in contrast to the models which incorporate satellite elevation parameter. The advantage of these models is their sensitivity to sudden unmodelled biases like ionospheric scintillation. Although models incorporating scintillation indices need further investigation, the initial results are promising with a performance superior to the one obtained with the elevation angle based models and comparable to the one obtained with the C/N0 model.

Based on these information next step of our research will involve research on detailed characterisation of residual observation errors, affected by ionospheric scintillation, which would enable to formulate an optimal stochastic model and a way of implementing this model into the RAIM algorithm. In order to design the stochastic model which best describes the true quality of observations one should analyse specific error sources in GNSS observations with particular emphasis on the influence of ionospheric scintillations.

Furthermore, further analysis will be addressed on carrierphase observations which are expected to be severely degraded by this phenomenon than code-phase one. The proposed approach will be also evaluated for the data affected by amplitude scintillations.

\section{ACKNOWLEDGMENTS}

The first author has been supported by the German Federal Ministry of Education and Research (BMBF) listed under the support code $16 \mathrm{~N} 036426$. Additionally, the authors gratefully acknowledge the contribution of the Department of Nautical Systems of the German Aerospace Centre for providing the necessary GNSS data, with which the present study has been performed.

\section{REFERENCES}

[1] M. Aquino, J. F. G. Monico, A. Dodson, H.; Marques, G. D., Franceschi, L. Alfonsi, V. Romano and M. Andreotti, "Improving the GNSS positioning stochastic model in the presence of ionospheric scintillation”, Journal of Geodesy, Vol. 83, pp. 953-966, 2009,

[2] J. Aarons, "Global Morphology of Ionospheric Scintillations", Proceedings of the IEEE, Vol.. 7O, No. 4, 1982

[3] W. Baarda, "A testing procedure for use in geodetic networks", Delft, Kanaalweg 4, Rijkscommissie voor Geodesie, 2, 1968

[4] J. Bartels, "The technique of scaling indices $\mathrm{K}$ and $\mathrm{Q}$ of geomagnetic activity”, Annals of the International Geophysical, Year 4, pp. 215-226, 1957

[5] S. Basu and K.M. Groves, "Specification and forecasting of outages on satellite communication and navigation systems", Space Weather, Geophysical Monograph, Vol 125, pp. 424-430, 2001

[6] S. Basu, K. Groves, S. Basu and P. Sultan, "Specification and forecasting of scintillations in communication/navigation links: current status and future plans”, Journal of Atmospheric and Solar-Terrestrial Physics, Vol. 64, pp. 1745-1754, 2002

[7] R. Brown, "Receiver autonomous integrity monitoring theory and applications”, in Global Positioning System: Theory and Applications, Volume II, Chapter 5, R. Parkinson, B. W. and Spilker, J. J. J. (Eds.), The American Institute of Aeronautics and Astronautics, Inc., V, Washington, D.C., USA, 559-59, 1996

[8] F.K. Brunner, H. Hartinger and L. Troyer, "GPS signal digfraction

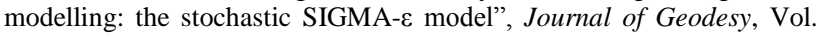
73, pp. 259-267, 1999

[9] W. Caspary, "Concepts of Network and Deformation Analysis” UNSW, Australia, School of Surveying, Monograph, 11, 1988

[10] R. Dach, U. Hugentobler, P. Fridez and Meindl, M. „Bernese GPS Software Version 5.0“, 2005

[11] A.J.V. Dierendonck, J.A. Klobuchar and Q. Hua, "Ionospheric Scintillation Monitoring Using Commercial Single Frequency C/A Code Receivers”, ION GPS-93, 1333, The Institute of Navigation, Arlington, 1993

[12] P.H. Doherty, S.H. Delay, C.E. Valladares and J. A. Klobuchar, "Ionospheric Scintillation Effects on GPS in the Equatorial and Auroral Regions”, Journal of The Institute of Navigation, Vol. 50, 2003

[13] H. Hartinger and F. Brunner, "Variances of GPS Phase Observations: The SIGMA Model”, GPS Solutions, Vol. 2, pp. 35-43, 1999

[14] C. Hegarty,M. B. El-Arini, T. Kim and S. Ericson, "Scintillation modeling for GPS-Wide Area Augmentation System receivers”, Radio Scienc, Vol. 36, pp. 1221-1231, 2001 
[15] K. R. Koch, "Parameter Estimation and Hypothesis Testing in Linear Models, 2nd. Edition”, Springer, Berlin, 1999

[16] R. B. Langley, “GPS receiver system noise”, GPS World, 1999

[17] B. Parkinson and P. Axelrad, "Autonomous GPS Integrity Monitoring Using the Pseudorange Residual", Journal of The Institute of Navigation, Vol. 35, pp. 255-274, 1988

[18] C.R. Rao, and H. Toutenburg, H., "Linear Models. Least Squares and Alternatives”, Springer, New York , 1995

[19] F. Rodrigues, M. Aquino, A. Dodson, T. Moore and S. Waugh, "Statistical Analysis of GPS Ionospheric Scintillation and Short-Time TEC Variations Over Northern Europe”, Proceedings of the 16th International Technical Meeting of the Satellite Division of The Institute

PE with Elevation Angle based weights

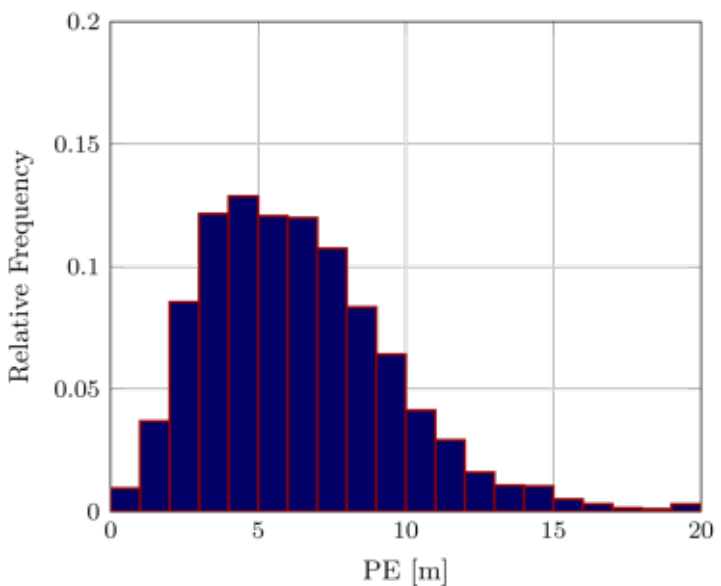

PE with Scintillation based weights

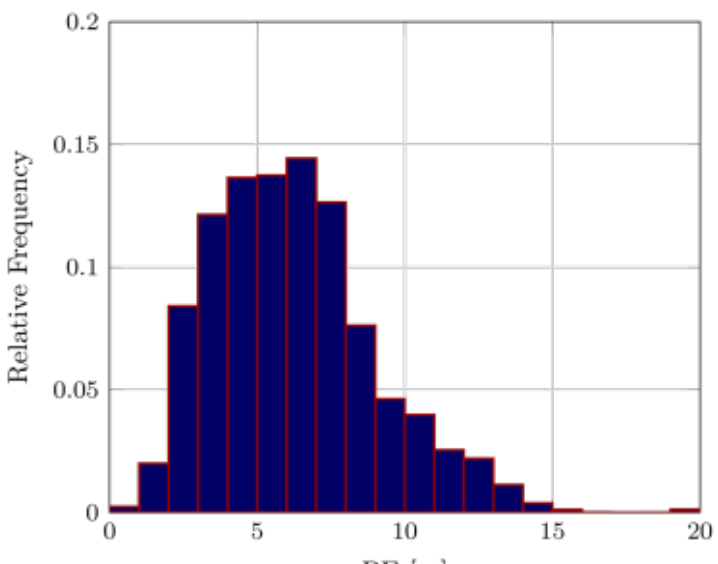

PE [m] of Navigation, Oregon Convention Center Portland, OR, September 912, 2003

[20] M. Rothacher, T.A. Springer, S. Schaer and G. Beutler, "Processing Strategies for Regional GPS Networks”, Proceedings of the IAG General Assembly (ed. Brunner, F.K), Rio de Janeiro, Brazil, 3-9 September 1997, IAG Symposia, 93-100, Springer Verlag, Berlin/Heidelberg/New York, 1998

[21] T. Walter and P. Enge, "Weighted RAIM for Precision Approach", Stanford University, 1995

[22] A. J. Wernik, J. Secan and E. Fremouw, "Ionospheric irregularities and scintillation”, Advances in Space Research, Vol. 31, pp. 971-981, 2003

[23] K.C. Yeh and C. H. Liu, "Radio Wave Scintillations in the Ionosphere", Proceedings of the IEEE, Vol. 70, No 4, 1982

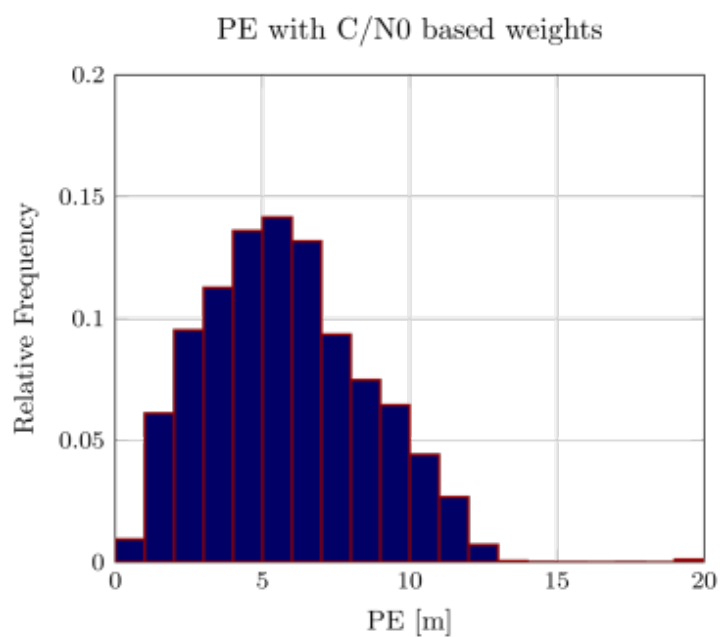

PE with Elev. Angle \& Scint. based weights

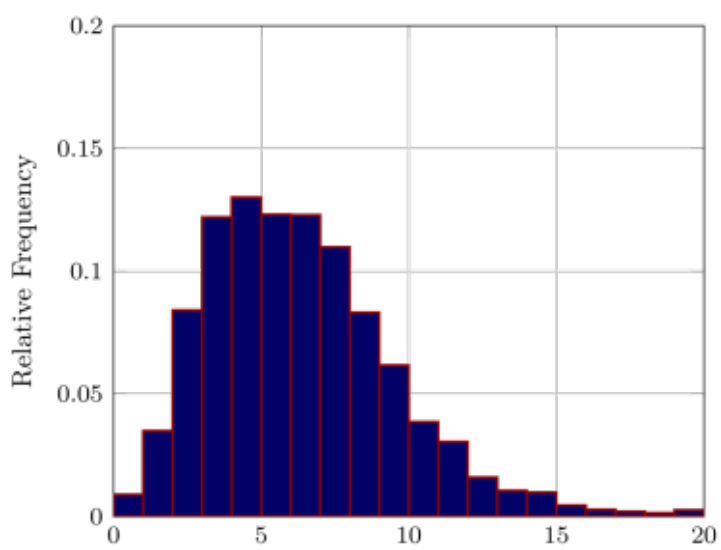

$\mathrm{PE}[\mathrm{m}]$

Fig. 9. Histograms of the weighted least-square solution 

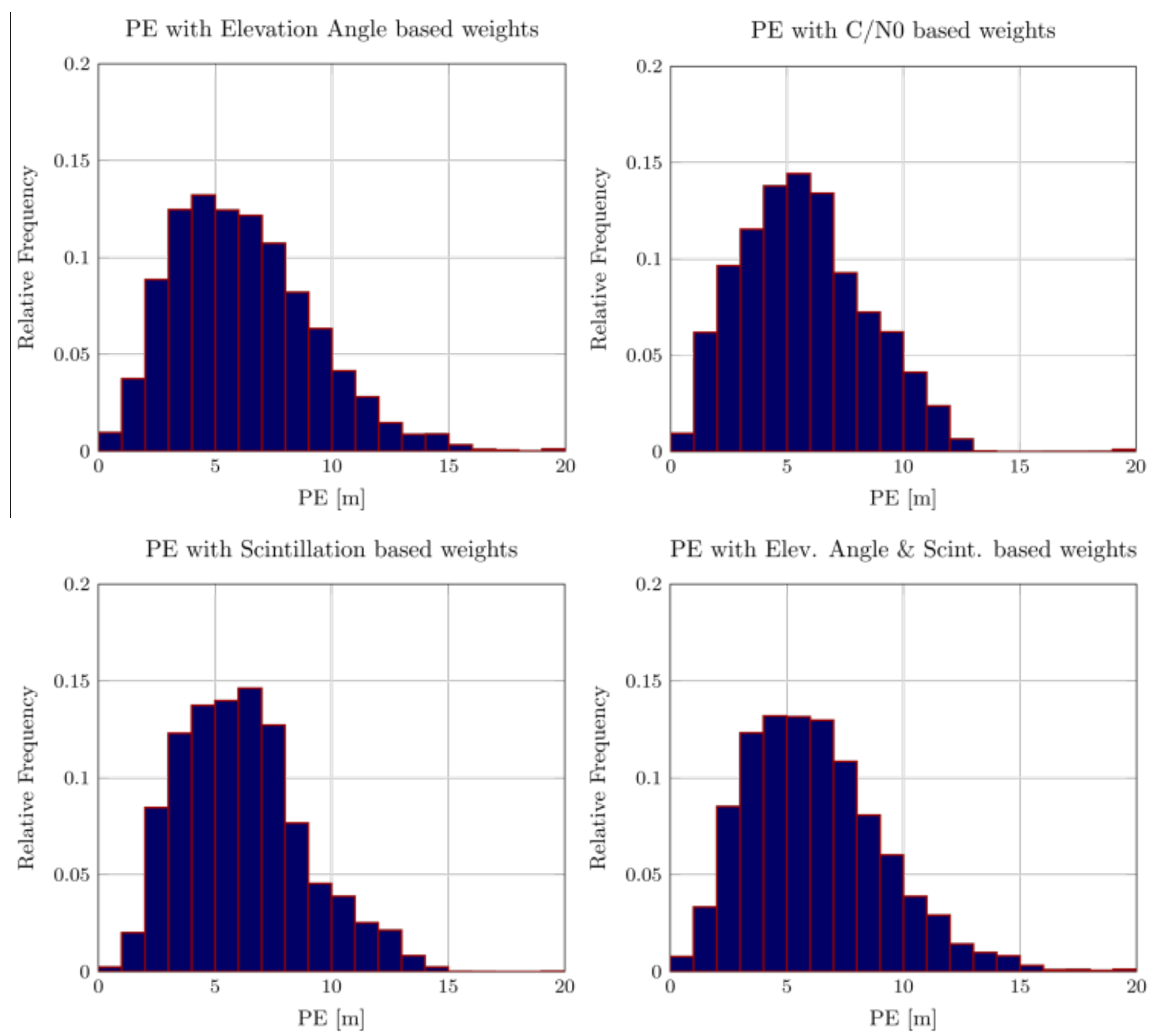

Fig. 10. Histograms of the weighted least-square solution with RAIM 